\title{
Neorectal Mucosal Prolapse After Intersphincteric Resection for Low-Lying Rectal Cancer: A Case Report
}

\author{
Mohammed Alessa ${ }^{1,2}$, Hyeon Woo Bae ${ }^{1}$, Homoud Alawfi ${ }^{1}$, Ahmad Sakr ${ }^{1}$, Fozan Sauri ${ }^{1}$, Nam Kyu Kim ${ }^{1}$ \\ ${ }^{1}$ Department of Surgery, Yonsei University College of Medicine, Seoul, Korea; ${ }^{2}$ Department of Surgery, College of Medicine, King Faisal \\ University, Al-Ahsa, Saudi Arabia
}

Radical resection for low rectal cancer is the mainstay among the treatment modalities. Intersphincteric resection (ISR) is considered a relatively new but effective surgical treatment for low-lying rectal tumor. As the sphincter preserving techniques get popularized, we notice uncommon complication associated with it in the form of rectal mucosal prolapse. We presented 2 rare cases that developed neorectal mucosa prolapse after ISR a complication following low rectal cancer surgery. Although ISR is a safe and effective surgical technique for low rectal cancer, it should be considered to correct modifiable possible risk factors. Also, Delorme procedure is good option for management of neorectal mucosal prolapse.

Keywords: Rectal prolapse; Intersphincteric resection; Low rectal cancer; Rectal neoplasms

\section{INTRODUCTION}

Radical surgery for low-lying rectal cancer is considered the main treatment modality among all modalities. Previously, some patients were the candidate of abdominoperineal resection (APR), but with the emergence of intersphincteric resection (ISR) anal sphincter can be preserved [1-3]. ISR is considered an effective surgical treatment for low-lying rectal tumors in selected patients [3-5]. As the sphincter preserving techniques get popularized, we notice uncommon complication associated with it in the form of rectal mucosal prolapse [6]. The symptoms of rectal prolapse include the feeling of a bulge or the appearance of reddish-colored mass that extends outside the anus. Other symptoms of rectal prolapse include pain and bleeding. It is also frequently associated with fecal incontinence [5].

Low anterior resection (LAR) syndrome such as frequency, urgency, and fecal incontinence is developed after taking down ileos-

Received: Nov 27, 2019 - Revised: Feb 21, 2020 • Accepted: Feb 22, 2020 Correspondence to: Nam Kyu Kim, M.D.

Department of Surgery, Severance Hospital, Yonsei University College of Medicine, 50 Yonsei-ro, Seodaemun-gu, Seoul 03722, Korea

Tel: +82-2-2228-2117, Fax: +82-2-313-8289

E-mail:namkyuk@yuhs.ac

ORCID: https://orcid.org/0000-0003-0639-5632

(C) 2021 The Korean Society of Coloproctology

This is an open-access article distributed under the terms of the Creative Commons Attribution NonCommercial License (https://creativecommons.org/licenses/by-nc/4.0) which permits unrestricted noncommercial use, distribution, and reproduction in any medium, provided the original work is properly cited. tomy. Besides, too redundant rectal mucosa sometimes begins to prolapse. Preoperative radiation and loss of internal anal sphincter with the frequent defecation might aggravate neorectal mucosal prolapse. In this article, we are reporting 2 cases of neorectal prolapse in patients who underwent ISR after ileostomy repair.

\section{CASE REPORTS}

This study was approved by the Institutional Review Board (IRB) of Severance Hospital, Yonsei University Health System (No. 4-2019-0987). The informed consent from patients were waived by IRB due to the nature of this study.

\section{Case 1}

A 64-year-old woman with body mass index (BMI) $20.57 \mathrm{~kg} / \mathrm{m}^{2}$ presented to the medical service with a history of hematochezia with constipation and change of stool caliber few days prior to her presentation. Upon investigation, she had normal baseline laboratory investigation but colonoscopy showed small anal lesion about $2 \mathrm{~cm}$ from the anal verge. She underwent transanal endoscopic mucosal resection, and the result revealed moderately differentiated adenocarcinoma with submucosal invasion and inadequate lateral resection margin.

She underwent robot-assisted LAR with total ISR, hand-sewn coloanal anastomosis, and diverting loop ileostomy. She had a smooth postoperative course and she was discharged home on 10th postoperative day in good general condition (pT1N0M0). 
Loop ileostomy was closed 3 months later. Eight months later, she developed neorectal mucosal prolapse manifested by wetting of underwear, anal mucosal bulge, and fecal incontinence (Wexner score, 20; Memorial Sloan Kettering Cancer Center [MSKCC] score, 57) (Fig. 1). Physical examination showed a short segment full-thickness mucosal prolapse. She underwent Delorme procedure (Fig. 2) for resection of prolapsed rectal mucosa and discharged a few days later in good health. The patient is on regular follow-up every 3 months and showed no mucosal prolapse (Wexner score, 18; MSKCC score, 52).

\section{Case 2}

A 57-year-old woman with BMI $24.9 \mathrm{~kg} / \mathrm{m}^{2}$ had a history of anal pain and bleeding per rectum for 2 months presented to the hospital for management. She underwent a colonoscopy that showed ulcerofungating mass of lower rectum, and biopsy resulted in adenocarcinoma. Magnetic resonance image for pelvis revealed cT3N1M0 rectal adenocarcinoma, $1.6 \mathrm{~cm}$ from the anal verge.

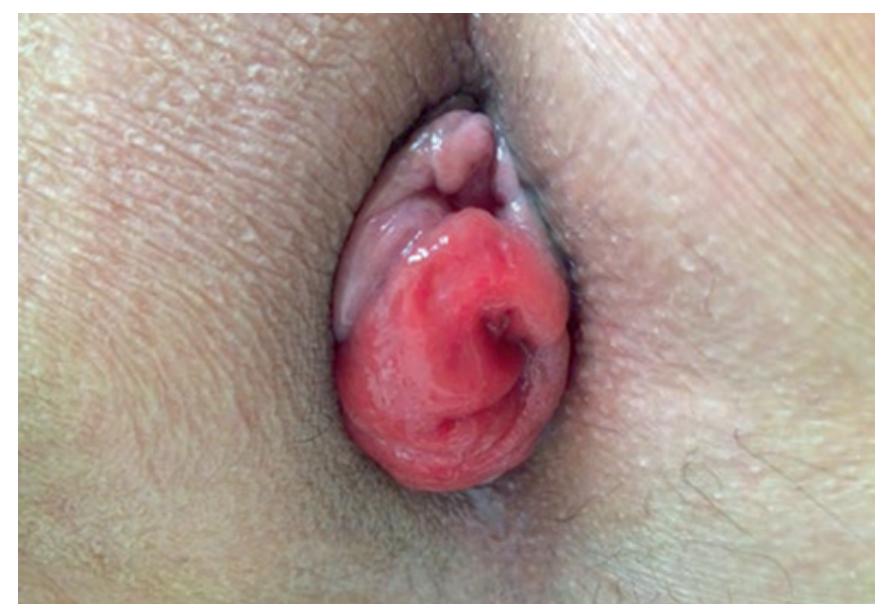

Fig. 1. Mucosal prolapse after intersphincteric resection for low-lying rectal cancer (case 1).
Staging computed tomography showed no distant metastasis. She underwent laparoscopic LAR with total ISR, hand-sewn coloanal anastomosis, and diverting loop ileostomy after long-course chemoradiotherapy. After 3 months, she developed rectal mucosal prolapse manifested by anal bulge and fecal incontinence. Physical examination showed short segment full-thickness prolapse. Two months later, ileostomy takedown and Delorme procedure done and the patient was discharged in good condition. Unfortunately, the patient lost to follow-up.

\section{DISCUSSION}

The incidence of rectal cancer is increasing among all population age groups, especially young age in Asian countries. Surgical resection remains the primary treatment modality for rectal cancer in addition to chemoradiotherapy. However, resection for low-lying rectal cancer is the most challenging in order to preserve anal sphincter and to avoid or minimize the incidence of APR in the era of increasing practice of sphincter preserving procedure. Secondary to that, we can notice uncommon complications related to this procedure. We are reporting 2 cases that developed neorectal mucosal prolapse a few months after LAR with ISR and coloanal anastomosis. After the literature review, we found few cases reported $[7,8]$.

Two cases in our study are not very old age considering common patient's age of rectal cancer and rectal prolapse (case 1,64 years old; case 2, 57 years old). Guraieb-Trueba et al. [6] showed that the average age of patients is 65.6 years in their cohort study of neorectal mucosal prolapse (19 patients), while Kuo et al. [9] reported 2 cases of average age 56.6 years. Based on reported cases, we noticed that it is common to occur in 5th and 6th decade. Also, Guraieb-Trueba et al. [6] found that it is more common in females (12 out of 19), and our reported cases are both female that is explained by a wide pelvis of females and weakness of pelvic floor muscles and laxity of ligaments due to pregnancy. Our 2 cases showed BMI of $20.57 \mathrm{~kg} / \mathrm{m}^{2}$ and $24.9 \mathrm{~kg} / \mathrm{m}^{2}$, while the
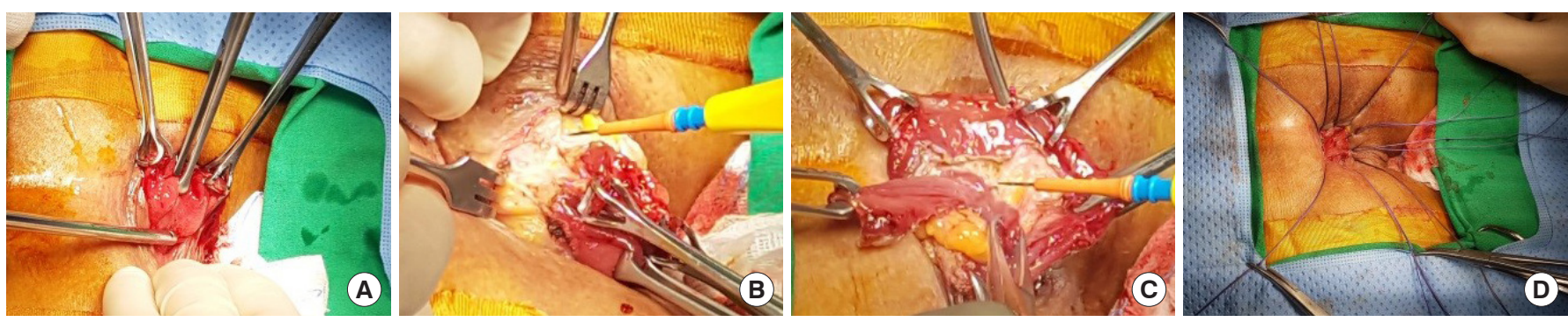

Fig. 2. It showed mucosal prolapse with different stages of Delorme procedure including dissection and anastomosis. (A) The patient was in prone position and digital rectal examination performed to identify mucosal prolapse. Then, grasping the mucosa of the rectum and prolapsed mucosa and submucosa to fullest extent with incision done along the scar of previous intersphincteric resection (ISR). (B) After that, circumferentially dissection of mucosa was started proximally. (C) Dissection was continued proximally at the point where redundant mucosa adequately removed. (D) Plication sutures was placed in muscular wall of the rectum. Lastly, redundant mucosa was resected and proximal mucosa sutured to the previous ISR incision line. 
previous study [6] reported average BMI was $24.2 \mathrm{~kg} / \mathrm{m}^{2}$. Many pieces of literatures described that obesity is a risk factor for rectal mucosa prolapse due to high pressure created by the bulky mesentery. However, it may not be such a meaningful in case of neorectal mucosa prolapse after ISR.

Case 1 was an early tumor that did not receive neoadjuvant therapy, while case 2 was stage III and received neoadjuvant. GuraiebTrueba et al. [6] showed that neoadjuvant chemoradiotherapy was given to all patients. By comparison with the nonprolapse group, they found a significant difference indicating that neoadjuvant chemoradiotherapy is a risk factor for the development of neorectal mucosal prolapse. In our study, however, the correlation was not distinct because neoadjuvant therapy was not done in 1 of 2 cases.

Our cases were operated by minimally invasive surgery (MIS) (case 1, robot-assisted; case 2, laparoscope-assisted), which is the same to other previous studies in that most cases were treated by MIS $[6,9]$. Prolapse can happen with all types of ISR, but it is more common with total ISR. Our 2 cases operated by total ISR [6]. Four cases of Yamada et al. [5] were also prolapses post total ISR. It is explained by increased intra-abdominal pressure postoperatively in addition to a thin neorectal wall in comparison with the rectum and loss of internal anal sphincter pressure. Although it is subjective, it is important to determine the optimal length of neorectum before doing coloanal anastomosis to avoid such complication $[5,6]$. In that way, it may be helpful to confirm the length if it is not too redundant and too tight by laparoscopy before and after anastomosis.

The presenting symptom of the 2 cases was wetting of underwear with a mucosal bulge that occurred after 8 months and 5 months after initial surgery for case 1 and 2, respectively. GuraiebTrueba et al. [6] reported that 4 patients $(21.0 \%)$ were diagnosed within 6 months of surgery, 8 (42.1\%) between 7 and 12 months, and 7 (36.8\%) over a year after surgery. Based on that observation, we noticed that symptoms most commonly start after 6 months up to 1 year. Our reported cases underwent Delorme procedure for the management of mucosal prolapse. We choose a transanal approach that had easy accessibility expecting dense adhesion due to previous surgery. On regular follow-up, the patient of case 1 did not show recurrence with little improvement of fecal incontinence (the patient of case 2 was lost to follow-up). Because postoperative fecal incontinence is commonly caused by ISR and radiotherapy, it can be difficult to achieve great results with surgery. According to Guraieb-Trueba et al. [6], 16 patients underwent transanal redo of coloanal anastomosis (Altemeier procedure) for neorectal prolapse and 1 of them experienced recurrence. Although Altemeier procedure showed a low recurrence rate, Delorme procedure also seems to be a good option in that it is less invasive and relatively simple. Besides, it is expected to show good results in the case of neorectal prolapse based on our experience. Further extensive study will be needed.

Lastly, from above mentioned data we can reach to some possi- ble risk factors responsible for neorectal mucosal prolapse. We have to be aware that this complication can be expected with those factors and keep in mind the need for further surgical intervention to decrease its incidence.

Although ISR is a safe and effective surgical technique for low rectal cancer, there is a risk of unusual complications such as neorectal mucosal prolapse. Commonly, the optimizing length of neorectum is focused on a tension-free anastomosis. However, too redundant neorectum also should be avoided because of this complication. Delorme procedure is a good and safe option for the management of neorectal mucosal prolapse.

\section{CONFLICT OF INTEREST}

No potential conflict of interest relevant to this article was reported.

\section{REFERENCES}

1. Heald RJ, Husband EM, Ryall RD. The mesorectum in rectal cancer surgery: the clue to pelvic recurrence? Br J Surg 1982;69:6136.

2. Kim NK, Kim YW, Min BS, Lee KY, Sohn SK, Cho CH. Operative safety and oncologic outcomes of anal sphincter-preserving surgery with mesorectal excision for rectal cancer: 931 consecutive patients treated at a single institution. Ann Surg Oncol 2009;16: 900-9.

3. Rullier E, Zerbib F, Laurent C, Bonnel C, Caudry M, Saric J, et al. Intersphincteric resection with excision of internal anal sphincter for conservative treatment of very low rectal cancer. Dis Colon Rectum 1999;42:1168-75.

4. Akagi Y, Kinugasa T, Shirouzu K. Intersphincteric resection for very low rectal cancer: a systematic review. Surg Today 2013;43: 838-47.

5. Yamada K, Ogata S, Saiki Y, Fukunaga M, Tsuji Y, Takano M. Long-term results of intersphincteric resection for low rectal cancer. Dis Colon Rectum 2009;52:1065-71.

6. Guraieb-Trueba M, Helber AR, Marks JH. Full-thickness neorectal prolapse after transanal transabdominal proctosigmoidectomy for low rectal cancer: a cohort study. Colorectal Dis 2018;20:5936.

7. Han JG, Wei GH, Gao ZG, Zheng Y, Wang ZJ. Intersphincteric resection with direct coloanal anastomosis for ultralow rectal cancer: the experience of People's Republic of China. Dis Colon Rectum 2009;52:950-7.

8. Akasu T, Takawa M, Yamamoto S, Ishiguro S, Yamaguchi T, Fujita $S$, et al. Intersphincteric resection for very low rectal adenocarcinoma: univariate and multivariate analyses of risk factors for recurrence. Ann Surg Oncol 2008;15:2668-76.

9. Kuo LJ, Ngu JC, Huang YJ, Lin YK, Chen CC, Tong YS, et al. Anorectal complications after robotic intersphincteric resection for low rectal cancer. Surg Endosc 2017;31:4466-71. 